\title{
Des petits jardins dans un " grand jardin »
}

Inventaire, étude et compréhension d'un phénomène jardinier furtif à Saulx-les-Chartreux (91)

Small gardens within a large one. Inventory, study and understanding of a surreptitious gardening phenomenon at Saulx-les-Charteux

\section{Antoine Quenardel}

\section{CpenEdition}

\section{Journals}

Édition électronique

URL : http://journals.openedition.org/ethnoecologie/2356

DOI : 10.4000/ethnoecologie.2356

ISSN : 2267-2419

\section{Éditeur}

Laboratoire Eco-anthropologie et Ethnobiologie

\section{Référence électronique}

Antoine Quenardel, «Des petits jardins dans un « grand jardin » , Revue d'ethnoécologie [En ligne], 8 | 2015, mis en ligne le 31 décembre 2015, consulté le 10 décembre 2020. URL : http://

journals.openedition.org/ethnoecologie/2356 ; DOI : https://doi.org/10.4000/ethnoecologie.2356

Ce document a été généré automatiquement le 10 décembre 2020.

\section{c) (i) $\Theta$}

Revue d'ethnoécologie est mis à disposition selon les termes de la licence Creative Commons Attribution - Pas d'Utilisation Commerciale - Pas de Modification 4.0 International. 


\section{Des petits jardins dans un « grand jardin »}

Inventaire, étude et compréhension d'un phénomène jardinier furtif à Saulx-les-Chartreux $(91)^{1}$

Small gardens within a large one. Inventory, study and understanding of a surreptitious gardening phenomenon at Saulx-les-Charteux

\section{Antoine Quenardel}

1 Situé à une quinzaine de kilomètres au sud de Paris, le secteur étudié s'inscrit dans un paysage préservé de l'urbanisation: 90 hectares environ, localisés sur la commune de Saulx-les-Chartreux, dans le département de l'Essonne (91), au piémont nord du Rocher de Saulx, dernier éperon du Plateau du Hurepoix.

2 L'absence d'urbanisation dans ce secteur rend particulièrement lisibles le relief et l'hydrographie ainsi que la situation d'entre-deux, entre plaine et coteau, espace ouvert et couvert, agriculture et boisement, ville et campagne. Entouré de toutes parts par « La France moche $»^{2}$, enchâssé dans l'agglomération, cerné par les infrastructures routières, situé dans l'axe des couloirs aériens de l'aéroport d'Orly, ce territoire a pu conserver ses qualités grâce à une volonté politique constante et forte.

Malgré les apparences et même s'il est tentant de l'imaginer, ce paysage préservé n'est pas intact. C'est un territoire dont l'occupation a évolué. Un regard attentif fait apparaître ça et là des parcelles en friches, des arbres fruitiers vieillissants, traces d'anciens vergers; en scrutant de plus près, on décèle également les signes d'une pratique jardinière furtive vivrière ou d'agrément - sur certains terrains. 


\title{
La politique locale et son combat pour le maintien de l'équilibre entre ville et campagne
}

\author{
Contenir l'urbanisation en protégeant et valorisant la forêt pour \\ l'ouvrir au public
}

4 La commune de Saulx-les-Chartreux s'est dotée très tôt d'outils de planification urbaine. Jean-Marc Bernard, maire entre 1959 et 1989 et conseiller général de l'Essonne, est architecte-urbaniste. Au fil des cinq mandats qui lui sont confiés, il mène avec son équipe une politique engagée et suivie en matière d'urbanisme. La volonté majeure de la commune est de circonscrire son urbanisation pour limiter l'étalement urbain sur son territoire. Cette position visionnaire a pour préalable et corollaire la protection de l'espace agricole. Un Plan d'Occupation des Sols (POS) est établi dès 1972. Dans les années 1970, le mitage du boisement par le bâti anarchique sur l'arête et le flanc orientés sud du Rocher, l'arrêt de l'exploitation des dernières carrières de sablon, la découverte d'un site géologique fossilifère majeur et les orientations du Schéma Directeur d'Aménagement et d'Urbanisme de la Région Île-de-France (SDAURIF) de 1976 poussent les pouvoirs publics à entreprendre prioritairement de protéger et de valoriser la forêt du Rocher de Saulx pour l'ouvrir au public.

5 Tout au long de cette période, c'est le caractère récréatif et les loisirs qui sont mis en avant dans les documents d'urbanisme régionaux. La forêt occupe la première place, le paysage et les milieux naturels font tout juste leur entrée en scène ${ }^{3}$ dans le projet urbain. La valeur de l'espace agricole n'est pas prise en compte. L'agriculture occupe tout au plus un rôle dans la gestion de l'espace.

\section{Reconnaître l'agriculture}

6 La Forêt Départementale du Rocher de Saulx, Espace Boisé Classé (EBC), est protégée au titre des Espaces Naturels Sensibles (ENS) depuis 1989. Dans les années qui suivent, le conseil général de l'Essonne poursuit sa politique en faveur de la protection des milieux naturels. À partir d'un recensement établi entre 1991 et 1993, les parcelles agricoles en friches (en particulier d'anciens vergers) situées en piémont nord du Rocher sont intégrées dans un périmètre de préemption foncière au titre des ENS afin de maintenir la diversité des milieux naturels et d'enrayer la régression des vergers. L'intérêt et la diversité écologiques de ces milieux proviennent du fait qu'ils ont été façonnés par l'agriculture et l'occupation humaine. Leur pérennité est conditionnée par un entretien minimum, mais peu d'actions concrètes suivent.

7 En 1994, le nouveau Schéma Directeur de la Région Île-de-France (SDRIF) inclut la plaine agricole de Saulx-les-Chartreux parmi les espaces à protéger dans la Ceinture verte et paysagère de la ville dense et centrale (Paris et sa proche banlieue) au titre des « Espaces agricoles de proximité ». Mais, de façon contradictoire, le SDRIF classe le nord du secteur cultivé en "Espace partiellement urbanisable». Dans un contexte où l'urbanisation grignote l'agriculture de manière particulièrement boulimique alentour, la grande vulnérabilité des terres agricoles ne cesse d'augmenter. Fermement opposée à l'annexion progressive de la plaine par la ville, la commune s'engage dans une politique véritablement active à partir de 1995, avec l'arrivée d'une nouvelle équipe à la mairie. La 
maîtrise du foncier et le maintien de la vocation des terres agricoles deviennent des priorités.

8 Une convention de veille foncière est signée en 1996 entre la commune et la Société d'Aménagement Foncier et d'Établissement Rural (SAFER). Elle débouche sur la mise en place d'un Périmètre Régional d'Intervention Foncière (PRIF) avec l'Agence des Espaces Verts (AEV) de la Région Île-de-France ; ce dispositif permet de maitriser l'attribution des parcelles et le maintien de leur vocation agricole, en recourant éventuellement à la préemption. Le POS de 1984 (révisé en 2000), puis le Plan Local d'Urbanisme (PLU) de 2012 vont délibérément dans le sens de la valorisation et de la protection des espaces naturels et agricoles. La commune fait valoir la valeur économique de cette activité (renforcée par les préoccupations d'approvisionnement des métropoles et la préférence donnée aux circuits courts), et son importance pour le maintien des espaces naturels, la qualité des paysages et son rôle dans la définition d'une identité.

9 La forte conviction sur le long terme et la détermination politique locale entraînent la création du Triangle Vert des villes maraîchères du Hurepoix en 2003. Son but est de maintenir et de développer l'agriculture périurbaine, au sein d'un projet de territoire intercommunal ${ }^{4}$. Cette dynamique contribue à infléchir le SDRIF en 2008 sur Saulx-lesChartreux ${ }^{5}$. En effet ce schéma directeur entérine et renforce les objectifs de la commune en considérant les limites de la plaine agricole comme un « front urbain d'intérêt régional ». Il souligne en outre la "vocation naturelle» du territoire, insistant sur les " continuités écologiques ou coupures d'urbanisation à maintenir ", les "continuités agricoles ou liaisons vertes à créer ou à renforcer»; enfin le Triangle Vert doit être respecté. La valeur de l'agriculture (en tant que telle et non plus comme mode de gestion ou réserve foncière) et des espaces ouverts devient une donnée à part entière du projet urbain.

10 L'attention portée aux milieux naturels et à leurs continuités est renforcée. Il est important de souligner le changement d'état d'esprit et le glissement qui s'est opéré en trente ans : la dimension récréative caractérisant l'approche des années 1970-1980, qui plaçait les loisirs du citadin au premier plan, est aujourd'hui reléguée à l'arrière-plan de préoccupations écologiques, paysagères et agro-sociétales nouvelles.

\section{Spécialisation - essor - déclin - déprise de l'agriculture maraîchère et fruitière dans la plaine de Saulx-les-Chartreux}

11 Il faut garder à l'esprit que la plaine agricole de Saulx-les-Chartreux, même si elle est aujourd'hui préservée, n'a cessé de se transformer. L'agriculture a connu, en un peu plus d'un siècle, une évolution rapide. Elle est passée successivement d'une agriculture de subsistance de type polyculture-élevage en mosaïque (céréales, élevage bovin, vigne, vergers et jardins vivriers), à une agriculture spécialisée (maraîchère et fruitière) en plein essor au début $\mathrm{du} \mathrm{xx}^{\mathrm{e}}$ siècle, puis au déclin successivement de l'arboriculture et du maraîchage dès la fin des années 1930. Incontestablement, le piémont nord du Rocher de Saulx a été affecté en premier par ces transformations. 


\section{0-1950 : un siècle d'essor maraîcher}

12 C'est avec l'urbanisation progressive et l'annexion d'une partie de son ancienne ceinture maraîchère par Paris que naît la vocation agricole de Saulx-les-Chartreux, à partir de 1800 et surtout autour de 1860. Les régions périphériques plus éloignées et les vallées du sud, dont celle de l'Yvette, s'organisent alors pour se saisir du marché d'approvisionnement en fruits et légumes de la capitale. Dans les années 1860-1870, la plaine de Saulx-les-Chartreux est le haut-lieu de productions renommées telles que le petit pois ou la fraise. Aux alentours de 1880, le phylloxéra décime les vignes; elles sont remplacées par des cultures de légumes de plein champ et de fruits. Les pommiers à cidre, cerisiers et poiriers abondent, particulièrement sur les pentes exposées au nord, là où la vigne ne se plaît pas; l'arboriculture fruitière constitue alors une activité complémentaire du maraîchage (Fleury \& Lenaers 2001).

\section{4-1937 : l'Arpajonnais, promoteur et transfuge de l'activité maraîchère}

13 La création de la ligne de tramway à vapeur l'Arpajonnais en 1894 est la conséquence du développement important de l'activité maraîchère sur le territoire. Le "train des légumes » dessert tous les villages du coteau du Hurepoix depuis Arpajon, et achemine les denrées directement au marché des Halles Centrales de Paris. Les exploitations agricoles, jusque-là tributaires des voitures à cheval, voient alors leurs fruits, légumes et fleurs expédiés de nuit, arriver directement aux Halles, entre minuit et quatre heures du matin. Lors des trajets de retour, la Compagnie des chemins de fer sur route de Paris à Arpajon (PA) approvisionne les cultivateurs en engrais et fumiers issus des écuries parisiennes. La ligne assure également un important trafic de voyageurs (main d'œuvre pour la cueillette de la fraise au mois de juin mais aussi promeneurs du dimanche) et du transport de messagerie (Fleury \& Lenaers 2001, Peyrafitte 1987).

Dans les années 1920, l'émergence des transports routiers par camion, d'usage plus souple car il évite les ruptures de charge, diminue l'importance du trafic de marchandise par chemin de fer. Accusée de retard, de lenteur, d'inconfort, de danger, de pollution et de nuisances, la ligne de chemin de fer, qui n'était plus rentable, ferme définitivement en 1937, remplacée par un simple service d'autobus pour les voyageurs. La disparition de l'Arpajonnais amorce le déclin de l'agriculture maraîchère de la région.

\section{Du recul à la déprise maraîchère}

La disparition progressive de la cavalerie parisienne au profit des véhicules à moteur après la Grande Guerre a pour conséquence de réduire l'offre en fumier de cheval destiné aux champs. Dans le même temps, la multiplication des usines d'incinération des ordures ménagères et des gadoues urbaines a pour but d'améliorer l'hygiène et de produire du chauffage urbain. Elle réduit fortement les tonnages d'engrais disponibles pour l'agriculture de banlieue.

L'utilisation de ces engrais urbains de proximité représentait un élément essentiel de la supériorité agricole de la banlieue et du secteur maraîcher, qui y recourait tout 
particulièrement. Il se trouve alors directement confronté aux difficultés d'approvisionnement (Phlipponneau 1956).

17 Avant la seconde guerre mondiale, l'activité maraîchère subit également la concurrence des régions méridionales et de l'Ouest (plaine du Rhône, du Roussillon, Bretagne) désormais reliées à Paris par des trains équipés de wagons isothermes (Fleury \& Lenaers 2001). En 1969, la suppression des Halles de Paris et la création du Marché d'Intérêt National (MIN) à Rungis facilitent un peu plus les échanges entre l'agglomération parisienne et des zones de production toujours plus éloignées.

À partir de 1950, la vente directe se développe au détriment de la vente en gros. Pour subsister, bon nombre d'exploitants s'équipent de camionnettes et vendent leur production sur les marchés forains essentiellement localisés au sud de Paris et sa banlieue. Le travail et le temps nécessaires à ce mode de commercialisation sont soustraits au temps passé dans les champs et la surface cultivée régresse proportionnellement.

19 Enfin, l'urbanisation galopante alentour laisse imaginer la disparition de l'agriculture à court ou moyen terme. Les maraîchers qui prennent leur retraite déconseillent souvent à leurs enfants de reprendre l'exploitation sous sa forme maraîchère, secteur où les tâches manuelles sont importantes et rudes, les revenus irréguliers. Les années 1980 confirment le déclin de l'agriculture maraîchère. Les cultures céréalières occupent de plus en plus d'espace sur la plaine et l'apparition des friches va en grandissant (Fleury \& Lenaers 2001).

\section{Le piémont nord du Rocher délaissé en priorité}

Les premières parcelles en friche apparaissent principalement sur les terres situées en piémont nord du Rocher, entre la route de Montlhéry et la lisière de la forêt du Rocher de Saulx.

21 Le sol, le relief et l'orientation sont les premiers ingrédients d'une désaffection. Sur l'ensemble du territoire agricole de Saulx-les-Chartreux, les terres cultivées, globalement limoneuses, sont très hétérogènes. Depuis toujours, les paysans se sont appuyés sur ces nuances pédologiques en affectant des cultures spécifiques à chaque nature de sol. Dans le nouveau contexte, les terres situées en lisière de la forêt de Saulx, de qualité moindre, en pente et orientées au nord, sont les premières à subir la déprise ; plusieurs parcelles sont délaissées, vendues ou converties prioritairement en jachères imposées par la Politique Agricole Commune (PAC) ${ }^{6}$.

22 Le découpage des parcelles en lanières constitue un deuxième facteur d'abandon. Dans le secteur étudié, le découpage de l'espace - conséquence de la combinaison entre un morcellement parcellaire important et un maillage dense de chemins ruraux - est plus prononcé que dans le restant de la plaine agricole. Il est vraisemblable que cela ait constitué une entrave à l'évolution de l'agriculture dans cette partie du territoire.

Le machinisme, amorcé discrètement en France après la Grande Guerre pour répondre au manque de bras et aux besoins urgents de reconstruction, s'intensifie après la seconde guerre mondiale. La culture des légumes qui nécessite une main d'œuvre importante et incompressible, les micro-parcelles en lanières et la petite propriété s'accommodent mal des techniques de production modernes qui tendent à simplifier le territoire. Le remembrement, mené à Saulx-les-Chartreux dans les années 1950, ne semble pas avoir 
d'effet majeur sur le découpage parcellaire du piémont nord ${ }^{7}$ comparé au reste de la plaine agricole, plus dynamique, qui connait, elle, des transformations notables.

Situé en périphérie du bourg, à l'écart des pôles et bâtiments d'exploitation, le secteur étudié présente des terrains de moindre qualité. Ils sont occupés par d'importantes plantations d'arbustes à petits fruits et d'arbres fruitiers isolés ou disposés en chapelets linéaires ${ }^{8}$, parfois imbriqués dans le boisement du Rocher. Le caractère secondaire de l'arboriculture fruitière au regard du maraîchage plus traditionnel, le manque de rentabilité (la cueillette des fruits est très consommatrice de main-d'œuvre), la dimension aléatoire de la production (due notamment au phénomène d'alternance dans la fructification), les difficultés relatives à la conservation et la maturation des fruits, la disparition du bouilleur de cru (vers le milieu des années 1980), l'éloignement des parcelles, l'augmentation du vandalisme, l'évolution globale de l'agriculture vers les grandes cultures et le déclin agricole généralisé : toutes ces évolutions ont démotivé les paysans et précipité l'abandon des parcelles plantées de fruitiers en priorité.

C'est là que persistent, encore aujourd'hui, les plus importantes traces de vergers, les arbres de la plaine ayant été pour la plupart arrachés. La quasi-totalité des témoignages recueillis auprès des jardiniers évoquent la présence d'arbres fruitiers, parfois très âgés, sur les parcelles acquises.

Entre 1979 et 1985, dans le cadre de l'opération d'aménagement du Bois du Rocher coordonnée par l'Agence Foncière et Technique de la Région Parisienne (AFTRP), le conseil général de l'Essonne entreprend d'acheter, à l'amiable et par voie d'expropriation, l'ensemble des parcelles privées non bâties situées sur le Rocher. Le but est de stopper l'urbanisation amorcée par mitage, d'aménager et d'ouvrir largement le site au public et de confier la gestion du boisement à l'office National des Forêts (ONF). Le périmètre de l'opération inclut plusieurs parcelles de verger enchâssées dans le bois qui, faute de gestion spécifique, sont rapidement gagnées par les ligneux forestiers et incorporées à ce qui devient la Forêt Départementale du Rocher de Saulx, plus tard classée au titre des ENS.

L'achat et le classement du bois contribuent à amenuiser un peu plus l'emprise agricole et en particulier celle des vergers - située au pied et en périphérie immédiate du Rocher. Il est vraisemblable que ce classement a joué un rôle en engendrant une forme de pression forestière accentuant par diffusion la déprise agricole et précipitant la vente de parcelles limitrophes situées au nord du piémont du Rocher.

\section{Des petits jardins dans un grand jardin}

Par la spécificité et la tradition maraîchère et fruitière de son agriculture, le Triangle Vert, et la plaine de Saulx-les-Chartreux en particulier, peuvent aujourd'hui encore, être comparés à un "grand jardin ". Ce territoire agricole de banlieue recèle aussi de multiples petits jardins aux origines diverses.

\section{Jardiniers des champs}

Le jardin paysan traditionnel est souvent dédoublé. Près de la maison, en prolongement de la cuisine, on cultive les productions précoces ou délicates que l'on souhaite avoir sous la main et qui nécessitent une surveillance et des soins assidus. Ce jardin, comme la basse- 
cour, est celui des femmes (Bergues 2011). Plus éloigné de l'habitation, et souvent en plein champ, se tient un autre potager, masculin celui-là. On y cultive des produits de longue conservation et des plantes fourragères de basse-cour. Ce type de jardin n'est pas toujours clos et souvent mal détaché de l'agriculture alentour (Corbin 1995). Ce deuxième potager labouré à la charrue n'est pas nécessairement fixe et il peut s'inscrire dans une logique d'assolement (Pluvinage \& Weber 1992). Traitées en jardins potagers, ces parcelles agricoles permettent ainsi de diversifier, ou au contraire de spécialiser la gamme des légumes que le cultivateur entend destiner à la vente, en fonction des goûts du paysan et de sa famille ${ }^{10}$. À Saulx-les-Chartreux, il est difficile de préciser quand, combien et quels terrains ont subi ce sort, mais on peut aisément imaginer que le déclin du maraîchage, conjugué avec le développement de la céréaliculture (inadaptée à l'autoconsommation), y ont fortement contribué.

30 À ces potagers paysans, s'ajoutent les parcelles agricoles héritées lors du partage de succession par les générations d'ouvriers ou d'employés qui succèdent aux maraîchers. Ces classes populaires d'origine rurale perpétuent l'attachement au jardin et la pratique du geste, ils ont à cœur d'entretenir ce qui subsiste du patrimoine foncier familial. On jardine par habitude, par culture et par besoin.

\section{Jardiniers des villes}

31 En France, la pratique de la villégiature est déjà répandue au XVIII ${ }^{\mathrm{e}}$ siècle chez les élites aristocratiques, qui se retirent l'été durant sur leurs terres, à la campagne. À partir de la fin du XIX ${ }^{e}$ siècle jusqu'au milieu des années 1950, chez nombre d'ouvriers et employés poussés vers la ville grise par l'exode rural, le « retour à la campagne » correspond moins à la découverte de la nature qu'au retour aux sources et aux origines familiales. Les «beaux dimanches" à la campagne leur font retrouver le grand air et le repos, l'idée d'une liberté perdue par l'asservissement d'une semaine de travail à l'usine (Corbin 1995, Dubost 1991). Le jardinage devient une façon libre de meubler le temps, un « délassement utile accessible à tout le monde » (Joigneaux 1865), qui permet aux milieux populaires d'acquérir le sens du loisir (Pluvinage \& Weber 1992). Faute de pouvoir aller à Deauville, Nice ou Biarritz, c'est en banlieue, accessible en transports en commun ou à vélo, que la classe populaire se rend en famille, entre voisins et amis, pour s'adonner aux plaisirs de la promenade, des jeux, de la java ou de la valse musette, des guinguettes et de la baignade... En même temps prolifèrent les jardins populaires, notamment dans l'entre-deux-guerres.

La ligne ferroviaire de Sceaux à l'ouest et l'Arpajonnais à l'est acheminent le dimanche nouveaux banlieusards et touristes, « artistes et excursionnistes désireux de respirer un air pur et de se récréer loin du bruit » (Pierre \& Helmic $1898: 19$ ), à une heure de Paris. Ils puisent dans le Guide Rose illustré: Paris-Arpajon, historique des localités desservies par cette ligne, édité en 1898, tous les renseignements utiles pour parcourir les alentours et en percer tous les secrets (horaires et tarifs des trains, histoire locale, commerces...). Les foires agricoles de la région (tomate, fraise, haricot) battent alors leur plein.

C'est durant cette même période que se développent les premiers lotissements privés sur les emprises d'anciens domaines ou bois. On pratique le jardinage-construction; cabanons et maisonnettes du dimanche sortent de terre au gré des moyens financiers et des économies des acquéreurs de terrains (Dubost 1991). Dans les années 1930, à Saulxles-Chartreux, le lotissement "Cottage artistique» des Rochers engage les milieux populaires citadins et les petites classes moyennes à investir dans un coin situé dans « la 
Petite Suisse à 18 kilomètres de Paris ». Il est aisément accessible le dimanche par l'Arpajonnais, par la ligne de chemin de fer Paris-Orléans ou la route Paris-Orléans (RN20). Sur une réclame racoleuse de 1930 on peut lire: "[Sa situation] rappelle les coquets aspects d'Alger ou les pentes abruptes du Massif Central. [...] Convient parfaitement aux gens de goûts artistiques ou rustiques, aimant jouir à $18 \mathrm{~km}$ de Paris, de la solitude et des charmes de la montagne ainsi que des joies des excursionnistes. [...] De l'air vif et pur qui convient aux anémiés et surmenés " ${ }^{11}$. "La banlieue c'est la santé » (Faure 1991) et ici, presque tous les ingrédients semblent réunis, air pur, horizon...

Cultivé et jardiné partiellement par des paysans ou des ouvriers fils de paysans locaux, ce territoire était déjà connu au début du XIX ${ }^{e}$ siècle de certains Parisiens, qui y trouvaient une destination dépaysante. Plus tard, quelques citadins des communes environnantes y voient un milieu accueillant. Plusieurs parcelles maraîchères et fruitières à l'abandon ou en déclin sont ponctuellement et progressivement converties au jardinage, parfois de façon éphémère. Au fil des années, le bouche à oreille opère. La mosaïque mêlant agriculture maraîchère et fruitière, maraîchage familial et boisements s'enrichit de potagers vivriers paysans et amateurs citadins, de jardins d'agrément. La taille réduite du parcellaire maraîcher, associée à la présence de l'eau ${ }^{12}$, rend le maraîchage et le jardinage compatibles, voire interchangeables.

Ainsi se crée au fil du temps un paysage riche en échelles d'espace (champ-jardin) et d'usages (travail-loisir) entremêlées. Agriculteurs et ouvriers salucéens, banlieusards des alentours ou parisiens, vivant dans d'anciennes habitations rurales, en lotissement pavillonnaire ou citadins en logements collectifs sans jardins, potageurs quotidiens, barbecuteurs du dimanche, amoureux de la nature ou les trois à la fois, actifs ou retraités... jardinent dans un paysage commun, lisière épaisse confortablement adossée au pied du Rocher.

La richesse des profils sociologiques des jardiniers rencontrés dans le cadre de l'enquête, la diversité des motivations, la foule de petites histoires collectées rendent compte de l'évolution de cette banlieue à chaque étape de son histoire autant qu'elles font écho à la grande histoire. Le déclin de l'agriculture maraîchère libère des petites parcelles qui fabriquent un territoire en attente, permissif, et produisent cette fresque modeste de jardins paysans, banlieusards et parisiens. À travers le jardin, le visage d'une banlieue hybride entre ville et campagne se dévoile. 
Figure 1 : « Jour de ma première communion, 17 juin 1945 »

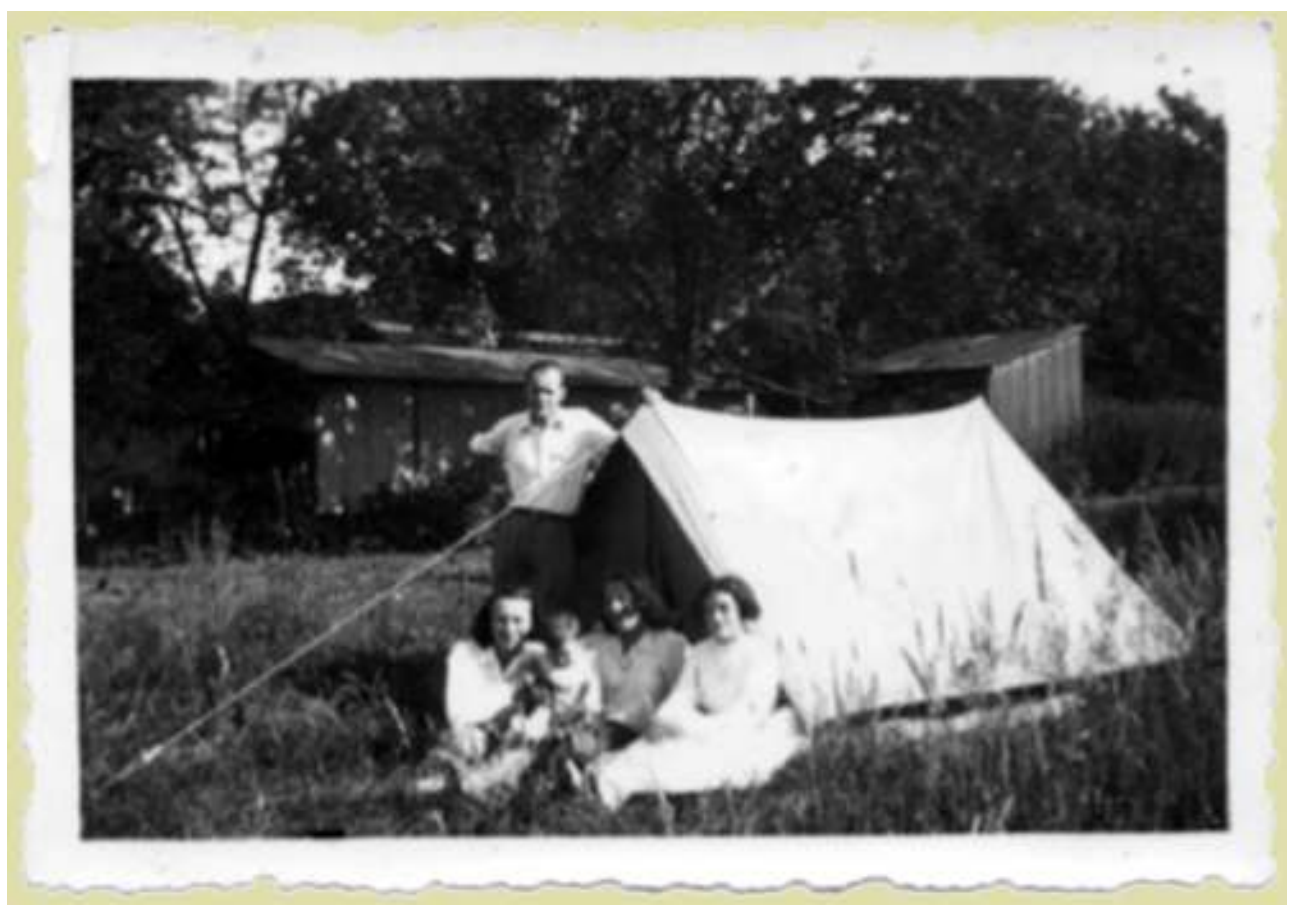

Crédits cédés à l'auteur

Figure $2:$ «C'était un jardin pour se reposer et s'évader, se ressourcer » (camping dans un verger, années 1930)

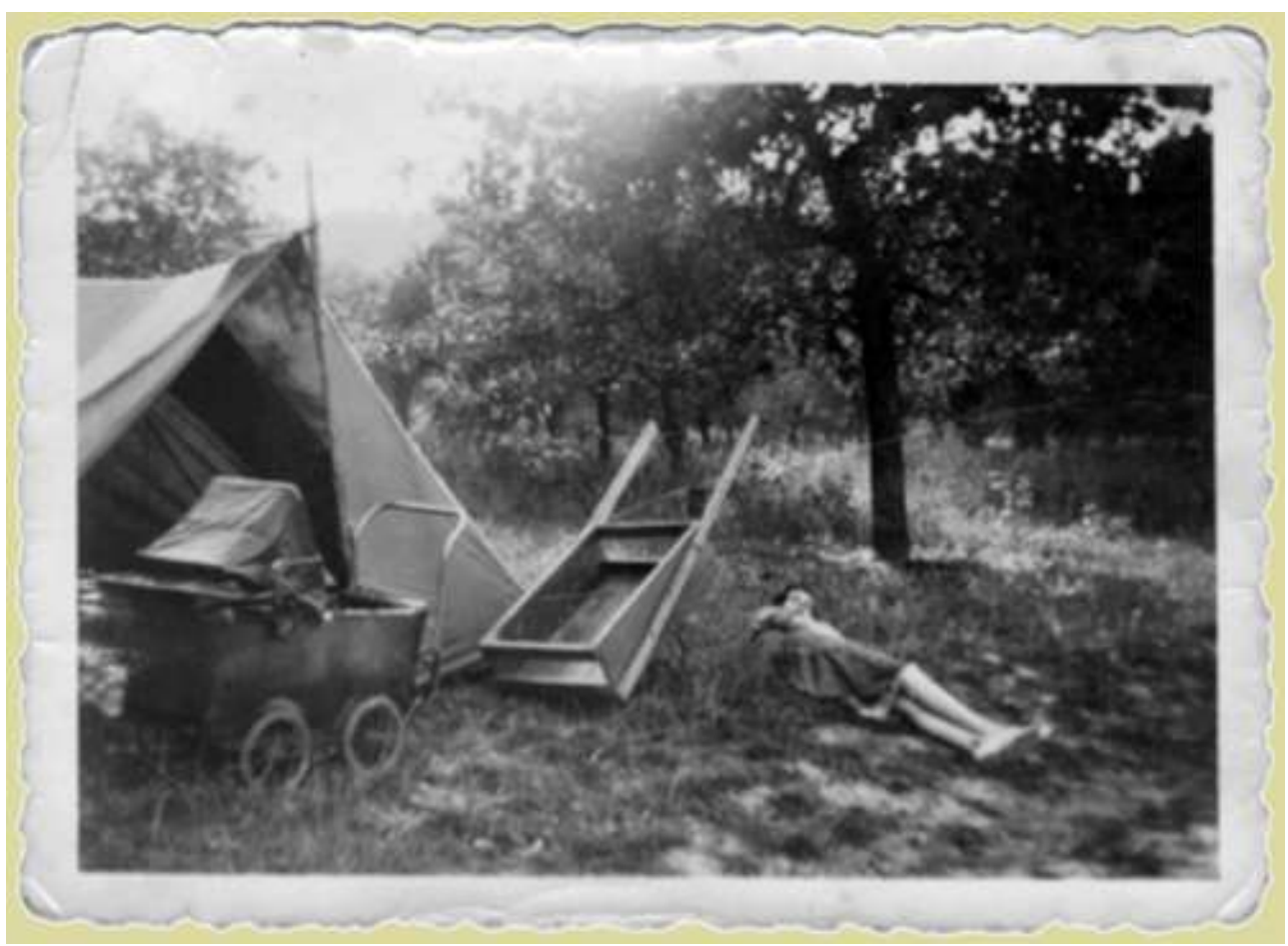

Crédits cédés à l'auteur

C'est au cours de promenades en piémont nord du Rocher de Saulx que des signes discrets témoignant d'une pratique jardinière sur certaines parcelles ont été repérés : reliquat 
d'une clôture grillagée en forêt, sapin de Noël quarantenaire dépassant d'un fourré13, puits oublié, planches recouvertes de mousses indiquant la présence passée d'un cabanon, strate arbustive horticole composée de cognassiers du Japon, seringats, forsythias sous le taillis de châtaigniers ou restes d'un bassin maçonné au milieu des bois...

Ces observations, mises en relation avec la présence de petits jardins potagers ou d'agrément toujours cultivés ou récemment abandonnés, ont été croisées avec les photos aériennes anciennes. Elles ont conduit à un inventaire de l'ensemble des parcelles jardinées, ayant été jardinées ou ayant pu être jardinées sur ce territoire, qu'on croyait exclusivement agricole. Au total, environ 130 parcelles ont été repérées. L'identification des propriétaires actuels, grâce aux données fournies par les services du cadastre, a permis de prendre contact ${ }^{14}$ avec les jardiniers initiateurs, repreneurs ou héritiers ${ }^{15}$ de ces jardins. Une quarantaine de témoignages ont ainsi pu être enregistrés et transcrits (soit $38 \%$ des courriers arrivés à destination). Un inventaire photographique et un herbier sont venus compléter ce corpus oral.

\section{Jardins non identifiés : l'ingrédient oublié du paysage}

L'espace étudié incarne parfaitement l'histoire de ce territoire et les grandes étapes de la politique de planification urbaine : presque totalement épargné par l'urbanisation, il est composé, en limite du relief boisé, de l'imbrication entre des espaces agricoles et des espaces naturels (souvent des friches consécutives à l'abandon de l'agriculture maraîchère et fruitière puis à celui du jardinage), classés respectivement $\mathrm{A}$ (secteur agricole) et $\mathrm{N}$ (secteur naturel) au PLU; ils correspondent aujourd'hui aux parcelles agricoles du PRIF et aux parcelles en friche du périmètre des ENS.

Étrangement, l'existence des jardins inventoriés - ni agriculture, ni espaces naturels n'apparaît nulle part ; ils ne font, par conséquent, l'objet d'aucune reconnaissance, ni au plan spatial ou paysager, ni au plan socioculturel, ni au plan économique. À peine évoquée dans un état des lieux, tout au plus tolère-t-on cette occupation sans statut, jugée mineure et considérée comme transitoire ${ }^{16}$. Les jardins n'entrent dans aucune des catégories ou prérogatives gérées par l'AEV et le Conservatoire des ENS, ils ne sont pris en compte dans aucun des projets territoriaux; leurs jardiniers non plus, la valeur du jardinage et les pratiques sociales associées encore moins.

41 Pourtant, cet ensemble de jardins fabrique une forme d'occupation du foncier qui, furtive et relictuelle au regard des autres grandes composantes du paysage, n'en demeure pas moins évidente au vu du nombre et de l'implantation cohérente des parcelles. L'étude réalisée démontre en outre le caractère historique de cette occupation, en relation avec le monde agricole et urbain.

Espaces de liberté pendant un temps, puis soumis à la réglementation et aux projets politiques conjugués au départ de certains jardiniers devenus trop âgés ou démotivés, ces parcelles de jardin subissent depuis une quinzaine d'années le même sort que les parcelles agricoles: déprise jardinière et enfrichement. Le soutien politique en moins. Sans volonté de maintien, les jardins, les jardiniers, la pratique du jardinage et les usages sociaux corrélés disparaissent, non seulement sous l'effet des dynamiques de reconquête naturelle par le végétal mais aussi des politiques appliquées, indifférentes envers ce phénomène méconnu, ou jugé mineur et sans importance. 
Manque d'acuité ou de curiosité ? Absence de reconnaissance et de statut ? La présence de ces jardins poserait-elle, en outre et de façon inavouée, problème? Attention danger: jardins?

\section{Jardin versus « zone $U$ »} l'image sale et le caractère « bidonville » associés aux cabanes de jardins souvent réalisées à partir de matériaux récupérés, préalablement stockés avant d'être réemployés. À Saulx, l'absence apparente de dimension collective présidant à l'implantation des jardins et l'environnement agricole et naturel ont peut-être contribué à construire chez certains une image inquiétante de ces constructions éparses, perçues comme difficilement maîtrisables et défendables sur le plan esthétique. De la même manière que les cabanons bricolés étaient jugés dissonants à côté des opérations urbaines de constructions neuves et lisses dans les années $1970^{19}$, des abris de jardin auto-construits ou préfabriqués déparent en piémont du Rocher de Saulx, dans un paysage que certains fantasment strictement campagnard, agricole et naturel... Intact.

La spéculation foncière qui a parfois été exercée ici par les communautés de gens du voyage sédentarisés sur des terrains agricoles achetés à prix fort en détournant la réglementation, a probablement contribué à alimenter l'idée et l'image négatives du mitage et de la cabanisation par amalgame d'idées reçues. 


\section{Jardins versus « zone $A$ »}

49 deux disciplines, les jardins inventoriés ne font l'objet d'aucune reconnaissance au plan agricole. La pratique du jardinage est-elle une activité agricole? On peut naïvement s'interroger sur ce qui différencie le jardinage de l'agriculture, tant du point de vue de l'espace (la surface notamment) que du statut ou de la pratique.

agre/agriculture : même ambition? On serait tenté de le penser si on considère le jardin potager vivrier et si on envisage ces deux activités pour leur caractère productif, à plus forte raison quand l'agriculture prend la forme du maraîchage. Jardinier et cultivateur produisent tous deux poireaux, tomates, choux, salades et pommes de terre... et au fond, quelle est la différence entre un jardin potager et une parcelle maraîchère sinon l'échelle, la relation au travail et la destination des légumes, fruits et fleurs produits? Plusieurs jardiniers interviewés, lorsque je les questionnais sur la manière dont étaient cultivées les parcelles voisines, ont laissé toutefois entrevoir une certaine forme de hiérarchie au sein de la fratrie présumée du jardinier et du maraîcher : professionnel au champ, amateur au jardin.

51 L'opposition n'est pas nouvelle. Sur le plan théorique les points de vue divergent. En 1865 Pierre Joigneaux ${ }^{20}$ pense $q^{\prime}$ ' « il convient d'établir une distinction claire entre les hommes qui s'occupent de la culture des légumes, et de ne pas confondre ceux qui font de la culture maraîchère avec ceux qui font de la culture potagère, bien que chez les uns comme chez les autres il s'agisse de produire des plantes pour la consommation de l'homme». Selon lui, les premiers sont de vils industriels avides de profits et les seconds, des amateurs appliqués faisant preuve de goût. Son contemporain Charles Naudin ${ }^{21}$, plus conciliant, écrit un peu plus tard : «Le jardinage est cette branche de l'art agricole qui a pour but la création et la culture des jardins. [...] On pourrait dire encore en moins de termes que le jardinage est de l'agriculture en petit, et que l'agriculture est du jardinage en grand » (Naudin 1898). 
Figure 3 : « Sa production, c'était monstrueux ! Il en donnait aux amis, aux voisins. C'était sa passion, son plaisir. Il était presque maraîcher. »

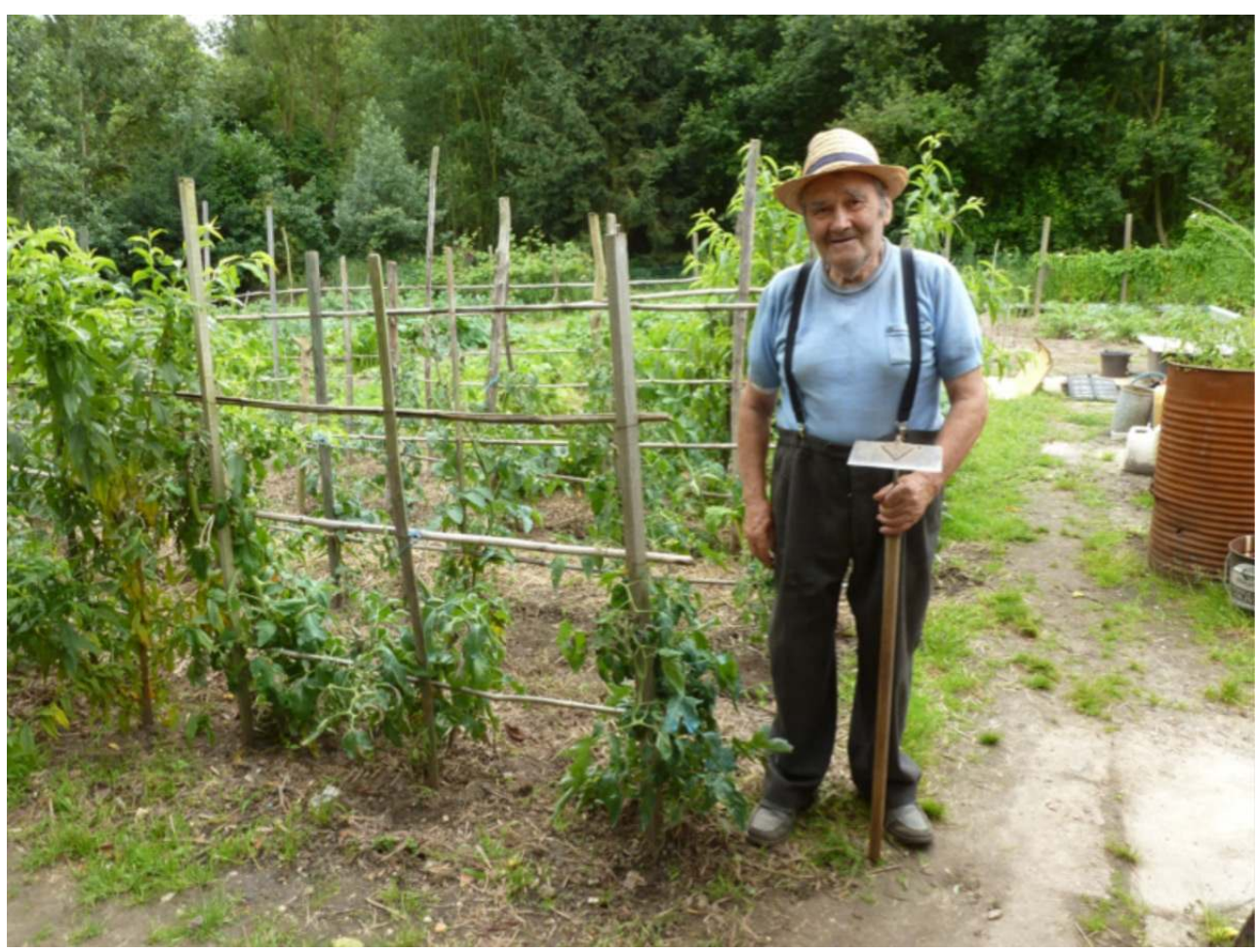

(c) A. Quenardel

Sur le plan commercial, dès la création des jardins ouvriers au XIX ${ }^{\mathrm{e}}$ siècle, les règlements intérieurs ont scrupuleusement veillé à interdire la vente des produits du jardin, s'opposant souvent à la pratique de la monoculture afin de ne pas tenter les jardiniers de concurrencer les exploitants maraîchers qui voyaient d'un mauvais œil ces amateurs souvent performants. La définition que donne le code rural de l'agriculture repose sur le caractère professionnel de l'activité (qu'elle soit exercée à titre principal ou non) et sur la finalité commerciale de la production (vente nécessaire des produits de l'exploitation) ${ }^{22}$. Ce n'est ni la technique ni la surface cultivée qui fait la distinction entre activité jardinière et activité agricole, mais plutôt le statut de celui qui cultive et la destination de sa production (autoconsommation familiale ou vente déclarée et contrôlée). Or, évaluer l'agriculture comme on le fait depuis plus de cinquante ans sur les rendements et la seule production matérielle de denrées marchandes est une approche aujourd'hui désuète ; elle ne prend pas en considération les nouvelles formes d'agriculture dites non productives et le caractère de plus en plus multifonctionnel de l'activité. D'ailleurs, du point de vue du régime social, le métier de paysagiste-jardinier ${ }^{23}$ est rattaché à la Mutuelle Sociale Agricole (MSA); le jardinier - professionnel - est donc considéré comme un agriculteur ; quelle production matérielle est issue de son exploitation?

Ces activités respectives sont en outre interchangeables et le statut des parcelles cultivées (pour l'agrément ou le profit économique) est réversible. On l'a vu, la totalité des jardins ont été mis en place sur des parcelles d'origine agricole. La terre y est travaillée, elles peuvent sans difficulté retourner à l'agriculture. Au-delà du caractère convertible d'une discipline à l'autre, c'est aussi la lutte contre la friche et l'établissement de la forêt qui rapproche les pratiques jardinière et agricole. Le Code rural impose à tous les 
propriétaires fonciers de terres agricoles la mise en valeur de leurs biens. Pour le législateur, ce qui compte, c'est le maintien, amateur ou professionnel indifféremment, de la valeur cultivable de la terre.

Enfin, il est amusant d'observer que le Triangle Vert participe aux journées annuelles «Jardins secrets, Secrets de jardins » organisées par le conseil général de l'Essonne, en proposant de faire découvrir à tous l'agriculture du territoire autour de l'idée que «les champs sont le jardin de la ville».

$55 \mathrm{Au}$ fond, malgré la filiation historique entre agriculture et jardinage amateur, si les jardins étudiés n'ont pas été reconnus et intégrés à la "zone A ", c'est peut-être parce qu'ils incarnent concrètement le déclin de l'agriculture qu'ils sont venus relayer, sans pour autant la chasser ou la menacer... Les parcelles de jardins inventoriés et étudiés, situées dans le PRIF, ne peuvent aujourd'hui plus être vendues à des jardiniers amateurs non agriculteurs susceptibles d'y mener des projets non agricoles.

\section{Jardins versus « zone $\mathrm{N}$ »}

56 La plupart des parcelles identifiées comme étant - ou ayant été - des jardins sont aujourd'hui comprises dans le périmètre des ENS qui identifie uniquement les espaces urbains, agricoles et naturels ainsi que les milieux humides. Elles seront préemptées et gérées à terme par le département de l'Essonne. Les écologues voient d'un assez mauvais œil l'occupation jardinée et la présence des jardiniers sur cette portion de territoire. En 2002, un rapport du bureau d'études Pierre Dufrène classe les quelques jardins potagers observés dans la rubrique des "unités écologiques artificielles» juste après les autoroutes, les décharges ou les grandes cultures céréalières (!) et préconise la résorption du bâti et du mitage que l'existence de jardin induit ${ }^{24}$. En 2012, le conservatoire des ENS s'y emploie via une campagne de «renaturation » des parcelles dont le département est propriétaire. Faire nature reviendrait ainsi à faire sans présence humaine installée (sans cabanes ni jardins). Jardin et jardinier ennemis de la nature, c'est un peu l'idée qui est ici sous-tendue. Une nature tout juste traversée, dans laquelle l'homme est tenu à distance, pour ne pas dire en dehors mais que le conservatoire des ENS se doit d'aménager et d'ouvrir au public.

Ce territoire est le fruit de siècles de présence humaine; il tire aujourd'hui en grande partie sa richesse et son potentiel écologique du façonnage dû à la main de l'homme. La présence d'une colonie de chouettes chevêches sur le territoire est la manifestation emblématique de cette coopération entre présence humaine et nature : cette espèce niche le jour dans les arbres creux des anciens vergers abandonnés; elle chasse et se nourrit la nuit dans la plaine agricole cultivée. Sa présence et son maintien sont conditionnés par l'activité humaine.

Depuis une vingtaine d'années, les pratiques d'entretien des espaces verts opposent ceux qui prônent résultat, importance de la production et visibilité du travail fourni, à d'autres, en forte augmentation ces dernières années, qui défendent en priorité qualité environnementale, respect des êtres vivants et santé des jardiniers. Les espaces verts municipaux, analysés par Gaëlle Aggéri et Pierre Donadieu (2003), sous l'angle de la gestion différenciée, ont fait les premiers l'expérience de ces évolutions conflictuelles. Si jardiner c'est vivre et faire vivre, c'est également, de plus en plus aujourd'hui, laisser vivre. Les théories et les écrits du paysagiste Gilles Clément (1994 et 1999) ont largement contribué à diffuser ce message auprès du grand public, des collectivités et des 
professionnels. Le «Jardin en mouvement » préconise un mode de jardinage qui va le plus possible avec et le moins contre les énergies et les éléments du vivant. Le concept du "Jardin planétaire» traduit la conscience et la responsabilité prise par l'homme, habitant, citoyen et passager sur la terre. environnement naturel (Paquot \& Younès 2010). On voit aujourd'hui se multiplier les expériences de réconciliation entre l'homme et la nature. La littérature savante et les magazines de vulgarisation pratique abondent dans ce domaine. "Un vigoureux militantisme associatif se réclame de l'unité du vivant, de la solidarité de destin entre les hommes, les animaux et les plantes, et entend sur ces bases requalifier l'ensemble du cadre de vie et restaurer le lien social» (Dubost \& Lizet 2003) : quel autre lieu que le jardin incarne mieux cette ambition?

60 Cultiver le sauvage, préserver et renforcer la diversité biologique, maintenir et enrichir la biodiversité alimentaire, cultiver l'humain... telles pourraient être les pistes d'un projet pour ce territoire en attente; afin de réconcilier l'homme et son environnement.

\section{Jardins sans importance : jardins sans valeur?}

$61 \mathrm{Au}$ cours des interviews, c'est en premier lieu l'accueil extrêmement positif et les réactions enthousiastes des jardiniers qui m’ont surpris. Les témoignages et les échanges sont ensuite venus confirmer cette impression. Ces jardins sont importants parce que leurs occupants le disent, et ils sont unanimes: le jardin - qu'il soit potager ou d'agrément - procure bien-être et bonheur.

Le jardin comme lieu de rencontre avec soi-même est aussi le lieu de la rencontre avec les autres. C'est un espace d'échange, intra et intercommunautaire, il est aussi l'endroit privilégié de l'acclimatation des gens venus d'ailleurs. Sociologues, ethnologues et historiens ont abondamment écrit sur ce sujet : le jardin populaire permet non seulement de se jardiner soi-même mais aussi de jardiner les autres (Cérézuelle 2003).

63 Les discussions avec les jardiniers démontrent que beaucoup d'entre eux manifestent une bienveillance particulière à l'égard du paysage partagé alentour bien au-delà des limites de leur parcelle. Outre l'attention qu'ils portent à la façade et aux abords immédiats de leur terrain, plusieurs jardiniers contribuent concrètement à l'entretien des chemins ruraux et des barrières d'accès. La présence humaine sur le territoire participe de surcroît du contrôle social, limitant incivilités et actes de vandalisme.

Depuis l'avènement du jardinage populaire, le jardin et son cabanon ne sont pas seulement les lieux de la pratique exclusive du jardinage et de la production des légumes. Ils servent souvent de support à d'autres activités (le bricolage, l'apiculture, la musique...) et de prétexte à bien d'autres usages sociaux : «Ce qui comptait, c'était moins le cabanon lui-même que l'espace autour, la possibilité de se retrouver à l'aise et au large. [...] Faire les fous, faire du bruit, rire et chanter : on ne risque pas de gêner les voisins comme dans les logements surpeuplés de Paris, on ne risque pas non plus la surveillance et les commérages de la parenté et du voisinage comme dans le village ou la bourgade de province. [...] Un espace sans contraintes, qui est aussi un espace de marge, aussi éloigné de la société urbaine que de la société rurale » (Dubost 1991). 
Figure 4 : « L'avantage qu'on a ici, c'est qu'on a notre terrain, on y fait ce qu'on veut. On n'a pas d'obligation. On est indépendant. On est vraiment libre. »

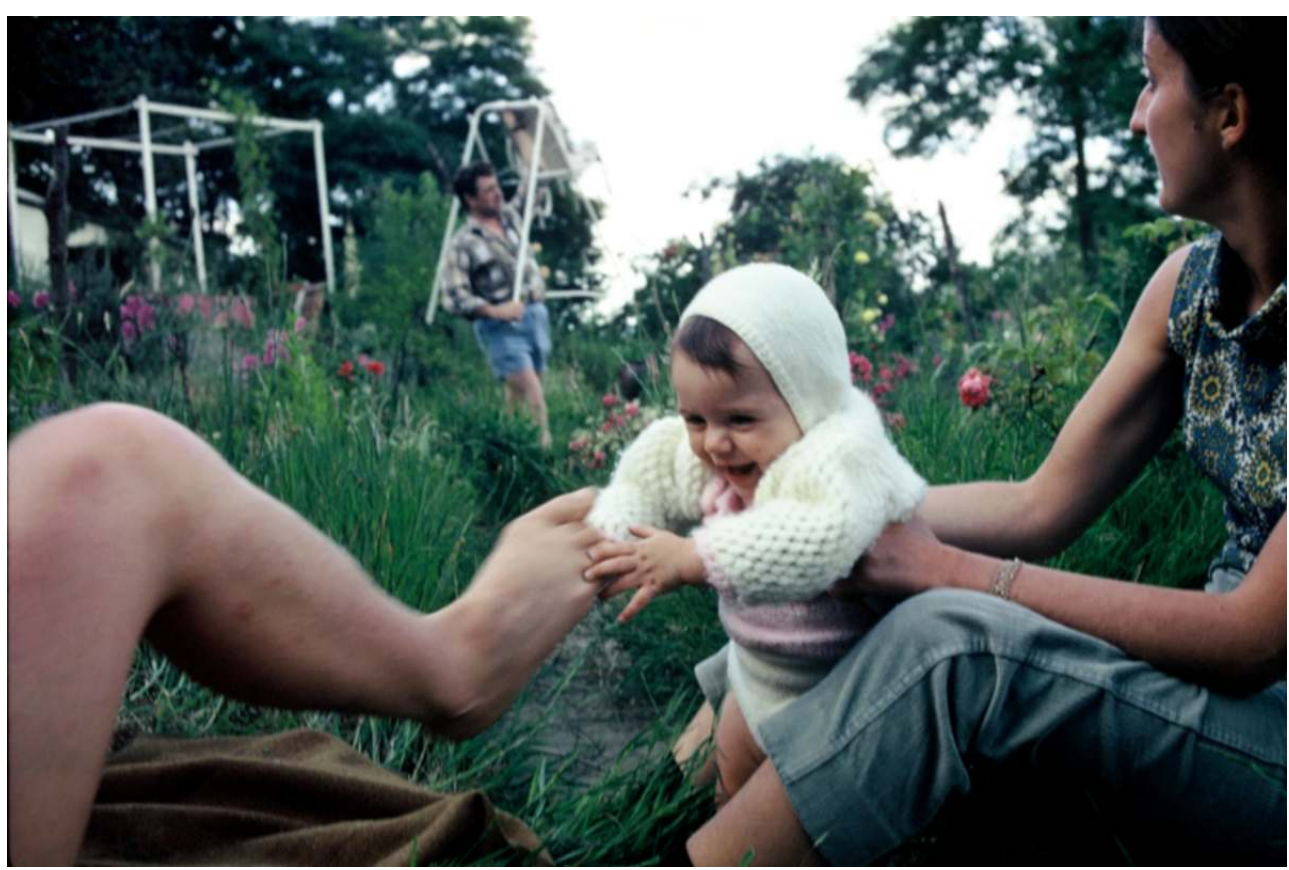

(C) A. Quenardel

Figure 5 : «Qu'est ce qu'on a pu faire comme fêtes au jardin ! Si vous connaissez quelqu'un qui veut s'en occuper, je ne lui loue même pas : c'est gratuit ! pour que ça continue à vivre... »

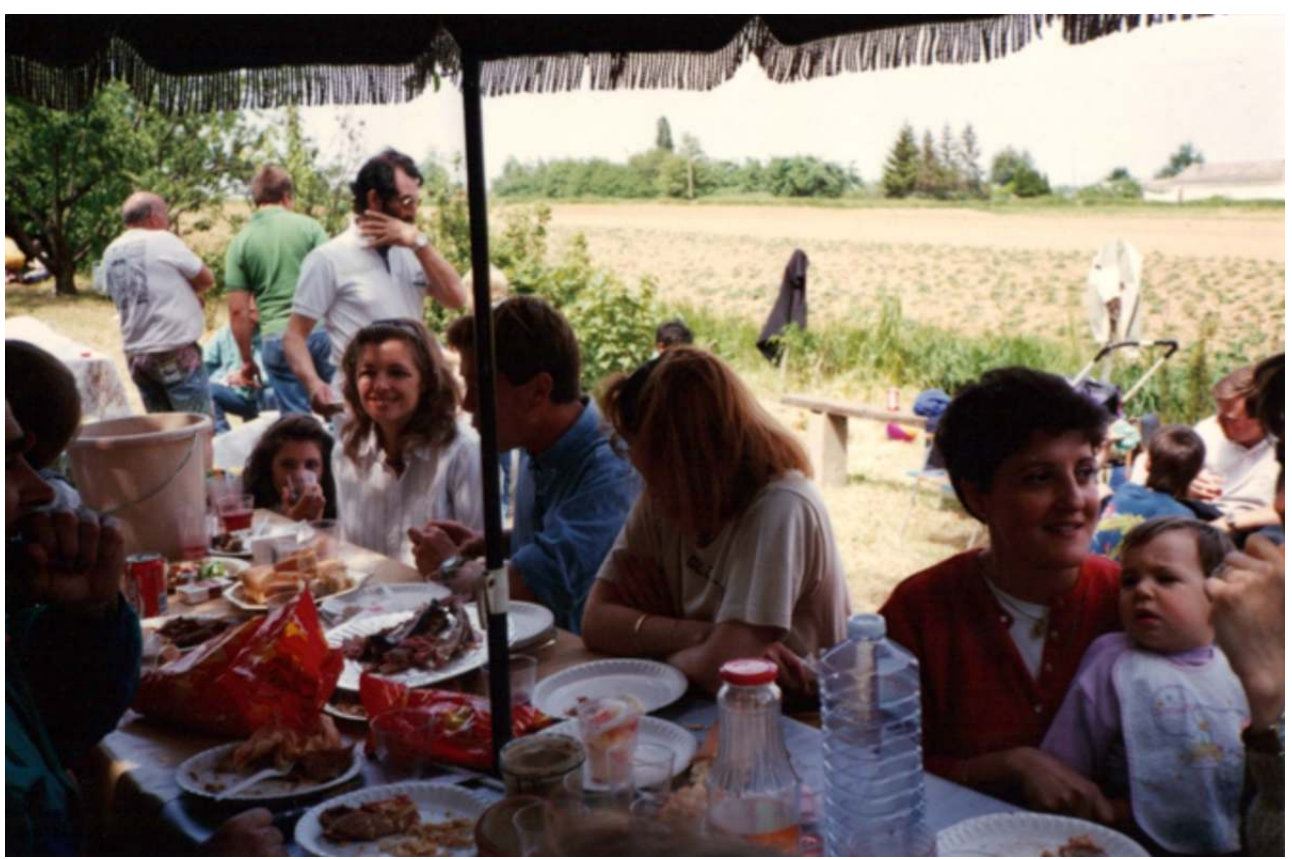

(c) A. Quenardel

65 Les jardins éloignés, comme ceux du coteau du Rocher à Saulx, sont des espaces à part, des endroits permissifs, des lieux de transgression. Espaces de villégiature dissociés de la résidence principale, ils offrent ce que l'habitat (qu'il s'agisse d'un appartement ou d'un 
pavillon) et le jardin (qu'il soit de village, de ville, de banlieue ou de lotissement) n'autorisent pas toujours. Des jardins comme des machines à fabriquer du possible.

Manuel Pluvinage et Florence Weber insistent sur l'importance et la valeur de la part du temps consacré à la pratique et aux productions des jardins populaires. Ils font part de son leur « admiration devant l'ingéniosité d'installations gratuites en argent et coûteuses en temps » (1992). On pourrait ajouter qu'elles sont inestimables en vécu, en sentiments et en souvenirs. Ce que permet de vivre le jardin (la part du réel), ce qu'il permet d'imaginer (la part du rêve), au plan individuel ou collectif, sont des éléments difficiles à quantifier. Or c'est peut-être là que se situe la véritable essence du jardin. Dans l'ensemble, les jardins étudiés puisent leur caractère vivrier en cela qu'ils aident à mieux vivre, visiblement. Mais comment mesurer cette dimension?

L'économiste Jean-Jacques Gouguet (1999), au sujet des jardins familiaux, propose de calculer leur valeur hors marché et d'examiner sous quelle forme on pourrait assurer la rémunération des services qu'ils rendent à la société25. Il s'agit de considérer l'ensemble des productions annexes relatives au jardin (santé, qualité du paysage, lien social...) ${ }^{26}$ et d'internaliser ces effets externes du jardinage dans les modes de calcul économique.

Cette approche reprend celle de Marx qui oppose à la coutumière "valeur d'échange " d'un bien ou d'un service, une "valeur d'usage ", c'est-à-dire sa valeur en fonction de l'utilité que le consommateur en retire. La valeur d'usage considère utile ce que la valeur d'échange envisage comme futile.

Dans la majorité des jardins étudiés, la pratique du jardinage a autant valeur d'usage que d'échange. C'est l'humain (les personnes, leurs proches, les autres) et son environnement que cultivent - ou cultivaient - les jardiniers dans leurs parcelles, au moins autant que les légumes, les fruits et les fleurs.

\section{Le saltus du Rocher de Saulx-les-Chartreux}

Situé entre la limite boisée du coteau et la plaine agricole cultivée, éloigné du bourg et des habitations le territoire où ils sont installés constitue une lisière épaisse, un entre-deux, un lieu à la marge qui, aujourd'hui encore, recueille pratiques et profils sociaux qui ne trouvent nulle part où aller. Périmètre aujourd'hui classé, en cours d'acquisition, c'est aussi un territoire en attente. Sachant que leurs terrains seront préemptés un jour, certains propriétaires de jardins ont cessé de les cultiver. Parallèlement, en l'absence de maitrise suffisante du foncier (probablement aussi de moyens?), le département de l'Essonne tarde à formuler un projet clair et rechigne à investir et gérer les quelques parcelles d'ores et déjà acquises. 


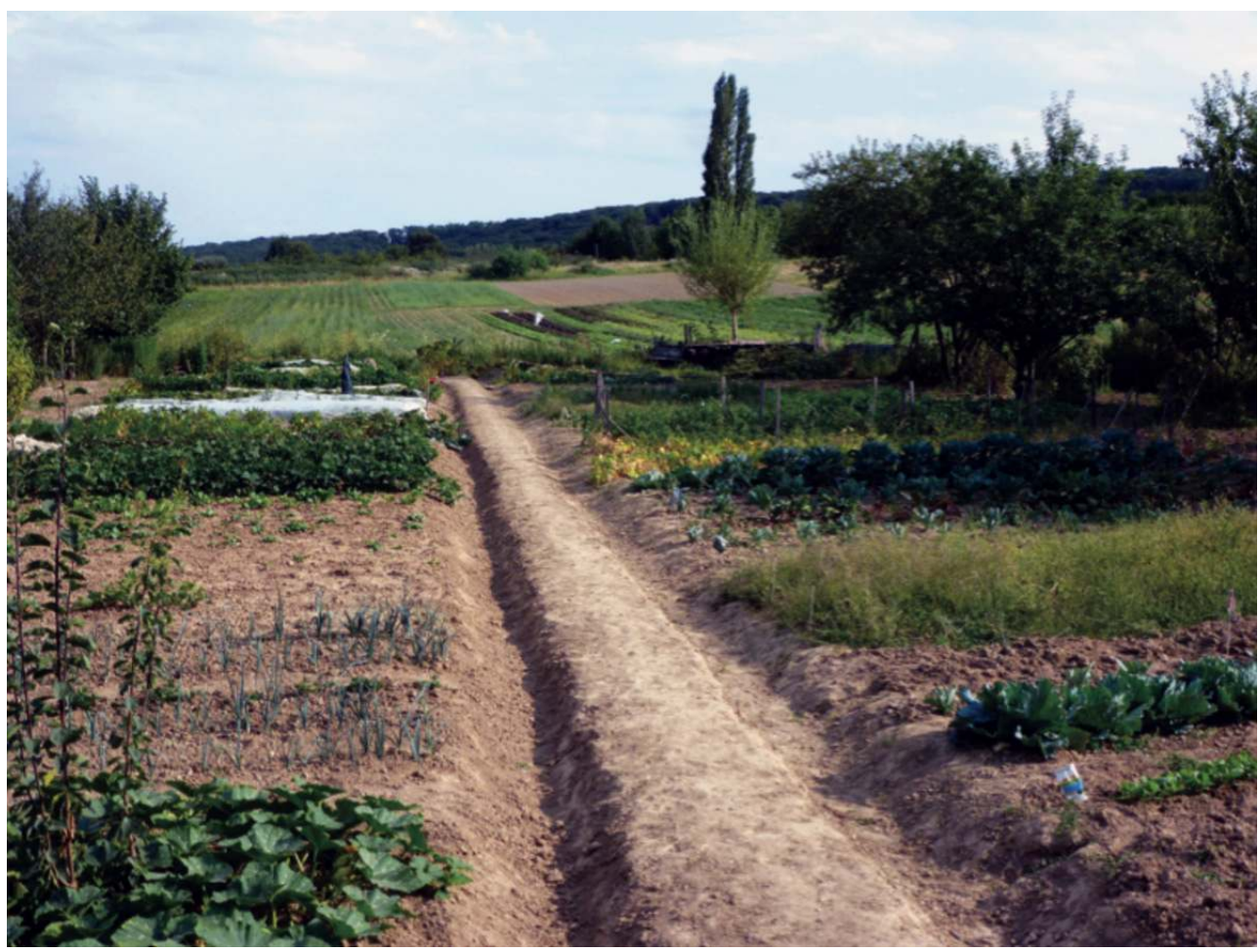

(C) A. Quenardel

71 En partie délaissé, le territoire d'étude entre dans la catégorie du Tiers paysage définie par le paysagiste Gilles Clément (2004). Dans la trilogie agraire conceptualisée par les agronomes romains, l'ager est défini comme l'ensemble des espaces labourés et cultivés et la silva comme les espaces boisés, tandis que le saltus est appréhendé par défaut, comme une catégorie intermédiaire. Ce sont les « terrains qui ne sont pas régulièrement cultivés et qui n'ont pas de couvert forestier continu et fermé », avec pour figure habituellement centrale, les parcours pastoraux. Cet espace de nature est utilisé par l'homme et constitue par conséquent une interface fonctionnelle et dynamique. Le géographe Georges Bertrand le décrit comme « un milieu écologique de transition, instable, menacé tour à tour par la mise en culture et la recolonisation forestière, et qui a enregistré toutes les fluctuations des sociétés et des économies rurales" (Bertrand 1975). À Saulx-les-Chartreux, les parcelles observées font figure d'avant-garde; elles expérimentent, finalement depuis longtemps, et en miniature, les principales aspirations mises en avant par le projet agrourbain du Triangle Vert : harmonie et solidarité entre ville et campagne, convivialité, lien social, bienveillance envers le territoire, circuits courts... C'est peut-être dans ces caractéristiques que réside, au fond, la véritable valeur du périmètre étudié : un espace au caractère impermanent et indéterminé. Un lieu accueillant sur le plan écologique, agroéconomique mais aussi social.

Le saltus du piémont du Rocher à Saulx-les-Chartreux est un tiers-espace mixte, entre agriculture, jardins et nature, un territoire mi-urbain mi-rural, un patrimoine vivant partagé... et à cultiver. 


\section{Épilogue (depuis 2012)}

73

Suite au travail réalisé, la position du conservatoire des ENS tend à s'assouplir. Impulsé par la communauté de communes Europ'Essonne, un groupe de travail sur le devenir des traces de vergers en friche situés en piémont du Rocher de Saulx a été constitué ${ }^{27}$. Le dialogue s'établit progressivement entre le département, la collectivité locale, les acteurs privés et associatifs concernés. Des propositions nouvelles et inventives articulant intérêt public et foncier privé sont en cours d'élaboration ; elles s'inspirent des expérimentations menées au parc départemental des Lilas à Vitry (94), périmètre classé ENS, des vergers familiaux mis en place à Besançon en 2011 ou des concepts défendus par les réseaux de partage de jardins entre particuliers ${ }^{28}$. La commune est en train de définir un périmètre de conservation des jardins sous certaines conditions; dans le cadre du programme «nature en ville» une convention cadre d'objectifs devrait être proposée par le conservatoire des ENS et signée entre les acteurs afin d'affirmer la vocation de petits jardins, à condition de ne plus utiliser de produits phytosanitaires, d'installer des clôtures légères végétalisées, de favoriser la plantation d'arbres fruitiers et de petits fruits rouges, pour peu à peu reconstituer un grand verger... jardiné !

\section{BIBLIOGRAPHIE}

Aggéri G. \& Donadieu P. 2003 - Le retour du sauvage dans les parcs publics. Les Carnets du Paysage 9-10:171-188.

Bergues M. 2011 - En son jardin. Une ethnologie du fleurissement. Paris, MSH, 400 p.

Bertrand G. 1975 - Pour une histoire écologique de la France rurale, l'impossible tableau géographique. In Duby G. \& Wallon A. (Ed.) Histoire de la France rurale, Tome 1. Des origines à 1340. Paris, Seuil : 8-118.

Cérézuelle D. 2003 - Les jardins familiaux, lieux d'initiation à la civilité. Communications 74 : 65-84.

Clément G. 1994 - Le jardin en mouvement - De la Vallée au Parc André Citroën. Paris, Sens \& Tonka, $101 \mathrm{p}$.

Clément G. 1999 - Le jardin planétaire. Réconcilier l'homme et la terre. Paris, Le Grand Livre du Mois, $127 \mathrm{p}$.

Clément G. 2004 - Manifeste pour le Tiers paysage. Paris, Sujet-objet, 69 p.

Corbin A. 1995 - L'avènement de la société des loisirs. 1850-1960. Paris, Aubier, 471 p.

Dubost F. 1991 - Le choix du pavillonnaire. In Faure A. (Ed.) Les Premiers banlieusards. Aux origines des banlieues de Paris, 1860-1940. Paris, Créaphis : 185-214.

Dubost F. \& Lizet B. 2003 - La nature dans la cité. De l'hygiénisme au développement durable. Communications $74: 5-6$. 
Faure A. (Ed.) 1991 - Les Premiers banlieusards. Aux origines des banlieues de Paris, 1860-1940. Paris, Créaphis, $283 \mathrm{p}$.

Fleury A. \& Lenaers V. 2001 - Diagnostic sur l'agriculture de Salx-les-Chartreux. Réflexion sur les stratégies communales. Rapport de recherche, Programme Agriculture urbaine, ENSP/INRA, 63 p.

Gouguet J.-J. 1999 - Jardins familiaux, croissance urbaine et insertion sociale : une analyse économique. In : Monédiaire G. Agri-cultures urbaines et ville durable européenne, droit et politique du jardinage familial urbain en Europe. Limoges, Pulim : 243-286.

Joigneaux P. 1865 - Conférences sur le jardinage et la culture des arbres fruitiers. Paris, Librairie agricole de La Maison Rustique., 139 p.

Naudin C. 1898 - Le potager. Jardin du cultivateur. Paris, Librairie agricole de La Maison Rustique, $178 \mathrm{p}$.

Paquot T. \& Younès C. 2010 - Philosophie de l'environnement et milieux urbains. Paris, La Découverte, $183 \mathrm{p}$.

Peyrafitte J. 1987 - Il était une fois l'Arpajonnais. Chemin de fer sur route de Paris à Arpajon, 1893-1936. Le Mée-sur-Seine, Amattéis, 202 p.

Phlipponneau M. 1956 - La vie rurale de la Banlieue Parisienne. Étude de géographie humaine. Paris, A. Colin, 503 p.

Pierre C. \& Helmic Y. 1898 - Guide Rose illustré : Paris-Arpajon, historique des localités desservies par cette ligne. Paris, Strauss, $220 \mathrm{p}$.

Pluvinage M. \& Weber F. 1992 - Les jardins populaires. Pratiques culturales, usages de l'espace, enjeux culturels. Rapport de recherche. Paris, Mission du Patrimoine ethnologique, Ministère de la Culture, 208 p.

\section{NOTES}

1. Une première version de ce texte a fait l'objet de mon mémoire de Master 2 - Jardins historiques, patrimoine et paysage - ENSA-Versailles/UP1 Panthéon-Sorbonne en 2012.

2. L'hebdomadaire Télérama du 10 février 2010 titrait «Halte à la France moche ", formule illustrée par une photo de la zone commerciale Villebon2 voisine.

3. Le secteur étudié passe du statut de Zone de transition à organiser dans le Schéma Directeur d'Aménagement et d'Urbanisme de la Région de Paris (SDAURP) en 1965, à Zone d'intérêt récréatif et/ou paysager et/ou écologique, inscrite dans la Frange des zones naturelles d'équilibre dans le SDAURIF en 1976.

4. Le Triangle Vert regroupe les communes de Marcoussis, Nozay, Villebon-sur-Yvette, Saulx-lesChartreux, Champlan et les exploitants agricoles motivés par le projet. Son but est de constituer une structure de réflexion et de communication entre municipalités, agriculteurs et usagers de l'espace. Le projet, qui s'appuie sur un diagnostic de territoire et un schéma directeur, propose un ensemble d'actions et de démarches pilotes.

5. Le document est invalidé par l'approbation du schéma d'ensemble du réseau de transports du Grand Paris en 2011. Il est corrigé et validé en 2013. Sur le secteur étudié, le SDRIF de 2013 conserve les principes de celui de 2008.

6. Entre 1988 et 2009, pour faire face à la surproduction céréalière, la PAC impose aux exploitations le gel obligatoire (improprement appelé jachère) de $10 \%$ des terres.

7. Certaines parcelles avaient déjà été délaissées ou cédées à des non agriculteurs avant et aussitôt après la guerre. 
8. Éloignées, ces cultures ne demandent pas une attention quotidienne comme les légumes et elles sont moins sujettes aux actes de vandalisme.

9. La formule est empruntée à Thierry Laverne, paysagiste et maire-adjoint de la commune de Marcoussis entre 1995 et 2014, co-fondateur du Triangle Vert.

10. Le Code rural entérine d'une certaine façon ce mode d'occupation à travers la notion de «parcelle de subsistance » : les maraîchers à la fin de leur carrière (propriétaires ou fermiers) ont le droit de poursuivre l'exploitation d'une partie des terres pour leurs besoins personnels et familiaux sans perdre leurs droits à la retraite.

11. Texte figurant sur une affiche-réclame imprimée (source: association d'histoire locale La Chartreuse Lyrique, section Histoire et Patrimoine.

12. Sources et rus abondent sur le territoire.

13. Le sapin de Noël, planté au jardin après les fêtes de fin d'année, n'est pas seulement un indice de la pratique jardinée d'une parcelle, c'est aussi le carbone 14 du jardin populaire permettant de le dater.

14. Lettre adressée par courrier puis relance téléphonique quand cela était possible.

15. La plupart des personnes interviewées sont retraitées. Ce sont parfois leurs parents - voire leurs grands-parents - qui ont initié un jardin sur la parcelle dont ils sont actuellement propriétaires ou indivisaires.

16. Les premières personnes rencontrées au début de cette étude (maire, adjointe à l'environnement de la commune et chargée de mission au Triangle Vert) semblaient perplexes quant à la matière à étudier, ces jardins n'existaient pas à leurs yeux.

17. Une exposition intitulée «Du cabanon au pavillon » a été organisée et présentée en 1994 par La Maison de banlieue et d'architecture d'Athis-Mons, 91.

18. Ces cahutes en gaules de châtaignier recouvertes de chaume permettaient aux cueilleuses de prendre leur repas à l'abri du soleil ou de la pluie et d'entreposer à l'ombre les paniers remplis.

19. À cette époque, pour subsister, de nombreux ensembles de jardins ont dû travailler le paraître, en harmonisant cabanes et clôtures.

20. Pierre Joigneaux (1815-1892), journaliste et homme politique d'extrême gauche, était agronome et enseignant.

21. Charles Naudin (1818-1899), biologiste et botaniste, fut enseignant et directeur du jardin botanique d'Antibes.

22. Paradoxalement, pour le législateur, le terme "exploitant» désigne sans distinction le paysan professionnel ou le jardinier amateur : décalage et incohérence.

23. Code APE 8130- $Z$ « service d'aménagement paysager ».

24. Par «bâti », il faut entendre des abris de jardin réalisés en auto-construction à partir de matériaux parfois synthétiques, mais presque toujours récupérés, donc recyclés.

25. À Londres par exemple, le dispositif Capital Growth, qui regroupe les jardins partagés en villes et l'agriculture urbaine, défend l'idée que la pratique du jardinage devrait pouvoir faire l'objet de prescriptions médicales et de remboursements par la sécurité sociale.

26. Voir Cérézuelle (2003).

27. Cela fait suite aux conclusions de l'audit sur les Trames vertes et bleues mené par le bureau d'études en écologie Biodiversita, qui désignait le piémont comme site d'actions prioritaires.

28. Voir par exemple les sites internet : www.plantezcheznous.com/, www.pretersonjardin.com/ 


\section{RÉSUMÉS}

Enchâssé dans l'agglomération, cerné par les infrastructures routières et dans l'axe des couloirs aériens de l'aéroport d'Orly, le territoire de petits jardins étudié, 90 hectares à une quinzaine de kilomètres au sud de Paris en fin du plateau du Hurepoix, a pu conserver ses qualités grâce à une volonté politique constante et forte. Préservé, ce paysage n'est toutefois pas intact, car l'occupation en a évolué. Un regard attentif fait apparaître des parcelles en friches, des arbres fruitiers vieillissants, traces d'anciens vergers, mais également les signes d'une pratique jardinière furtive, vivrière ou d'agrément. La véritable valeur du périmètre étudié réside dans son caractère impermanent et indéterminé. C'est un lieu accueillant sur le plan écologique, agroéconomique et social. Le saltus du piémont du Rocher à Saulx-les-Chartreux est un tiers-espace mixte, entre agriculture, jardins et nature, un territoire mi-urbain mi-rural, un patrimoine vivant partagé et à cultiver.

Set within the town and its suburbs, surrounded by road facilities and lined up with the orly airport air routes, the territory of the small gardens studied here covers 90 hectares and is situated about $15 \mathrm{kms}$ South of Paris, at the end of the Hurepoix Plateau; this territory managed to keep its qualities thanks to a constant and strong political will. This landscape is preserved, but not intact however, for its occupation has changed. If you look closely, you will see uncultivated plots, ageing fruit trees, traces of old orchards, but also signs of furtive, subsistence or pleasure gardening. The real value of the area studied here lies in its non-permanent and undetermined character. It is a welcoming place from an environmental, agro-economical and social point of view. The saltus of the piedmont plain of the Rocher at Saulx-les-Chartreux is a mixed third -party area between agriculture, gardens and nature, a half-urban half-rural territory, a living heritage which is shared and needs to be cultivated.

INDEX

Mots-clés : Jardins, agriculture, nature, politique publique, tiers espace, rural, urbain

Keywords : Gardens, agriculture, gardening, vegetable gardening, public policy, third space rural, urban

\section{AUTEUR}

\section{ANTOINE QUENARDEL}

Paysagiste DPLG et historien des jardins 